\title{
Emphysematous pyelonephritis with Dengue hemorrhagic fever: A successful medical management of a fatal scenario
}

\author{
Robin George Manappallil', Prathap Reddy Muthyala ${ }^{2}$ \\ ${ }^{1}$ Consultant-Physician, ${ }^{2}$ Resident, Department of Internal Medicine, Baby Memorial Hospital, Calicut, Kerala, India
}

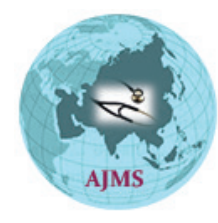

A B S T R A C T

\begin{abstract}
Emphysematous pyelonephritis is a life threatening acute necrotizing infection with associated gas formation involving the renal parenchyma and perinephric tissues. Dengue fever, according to the $\mathrm{WHO}$, is the most rapidly spreading mosquito borne viral infection in the world. Dengue Hemorrgahic Fever is a more severe form of dengue fever and is characterized by fever, bleeding manifestations, plasma leakage and thrombocytopenia. This is a case of a 56 year old female, who presented with history of fever, headache, myalgia and dysuria; and was diagnosed to have emphysematous pyelonephritis with Dengue Hemorrgahic Fever. She was successfully managed with intravenous antibiotics and platelet transfusions, without any urological interventions.
\end{abstract}

Access this article online

Website:

http://nepjol.info/index.php/AJMS DOI: 10.3126/ajms.v8i5.17847 E-ISSN: 2091-0576 P-ISSN: 2467-9100

Key words: Emphysematous pyelonephritis, Dengue fever, Dysuria, Thrombocytopenia

\section{INTRODUCTION}

The WHO has defined Dengue fever (DF) as an acute febrile illness of 2 to 7 days duration in the presence of two or more of the following features: headache, myalgia/arthralgia, retro-orbital pain, rash, haemorrhagic manifestation (petechiae, positive tourniquet test) and leucopenia. Dengue Haemorrhagic Fever (DHF), a severe form of DF, is defined as an acute febrile illness with one or more of the following features: petechiae/ecchymosis/ purpura, bleeding from mucosa/injection sites/other sites, hematemesis/melena, thrombocytopenia, evidence of plasma leakage and positive tourniquet test. Platelet and red cell transfusions are required in case of massive bleeding. ${ }^{1}$

Pyelonephritis refers to symptomatic infection of the kidneys. Mild pyelonephritis may present as low grade fever with or without low back pain; while severe cases manifest with high grade fever, chills and rigors, nausea, vomiting and loin pain. Emphysematous pyelonephritis (EPN), a more severe form of pyelonephritis, is characterized by necrosis and gas formation in the renal and perinephric tissues. It is commonly seen in diabetic patients. ${ }^{2}$ Antibiotic therapy along with immediate nephrectomy forms the mainstay of treatment. Recent advances also allow percutaneous drainage along with broad spectrum antiobiotics. ${ }^{3-5}$

\section{CASE REPORT}

A 56 year old lady, homemaker, presented to Medicine OPD with complaints of fever, headache and myalgia of 5 days duration. She also had dysuria for the past 2 days. She is a diabetic on insulin therapy. On examination, she was conscious, oriented and febrile $\left(101^{\circ} \mathrm{F}\right)$. Her blood pressure was $90 / 60 \mathrm{mmHg}$, pulse $110 /$ minute, respiratory rate $22 /$ minute with saturation of $94 \%$ (in room air). Her systemic examinations revealed a mild left sided loin tenderness.

Her blood investigations showed leucocytosis $(18,700$ cells/cumm) with neutrophilia (N96 L4), thrombocytopenia $(69,000$ cells/cumm), deranged renal functions (urea $61 \mathrm{mg} / \mathrm{dl}$ and creatinine $1.6 \mathrm{mg} / \mathrm{dl}$ ) and elevated liver enzymes (SGOT 206 and SGPT 268). Dengue NS1 antigen was reactive. Urine microscopy showed numerous pus cells. Other investigations like Weil Felix, 
leptospirosis serology, malarial card test, HIV, $\mathrm{HBsAg}$ and anti HCV were negative. She was started on injection cefaperazone and normal saline, and other supportive drugs like pantoprazole, paracetamol and folic acid. Her HbA1c was elevated and blood glucose levels were controlled with insulin.

The following day, she continued to be febrile and developed hematuria. Her platelet counts dropped to 12,000 cells/cumm and activated partial thromboplastin time was mildly prolonged (test 40 seconds and control 20 seconds). PT/INR was normal. Ultrasound abdomen showed ill defined, hypoechoic area with streaks of air in the upper pole of left kidney with thin rim of perinephric fluid, suggestive of EPN; which was confirmed on CT abdomen as Class 2 EPN (Figure $1 \mathrm{a}$ and b). Cefaperazone was stopped and intravenous meropenem was started (500 mg q8h). Platelet, RBC packed cells and fresh frozen plasma transfusions were given along with intravenous tranexamic acid. Urological interventions were avoided in view of thrombocytopenia. By day 4 of admission, her hematuria started decreasing and platelet counts started rising. Her urine culture grew Klebsiella, which was sensitive to meropenum. She was discharged of day 10 of admission in stable condition with normal complete blood count. Her repeat urine microscopy did not show any pus cells or RBC.

\section{DISCUSSION}

EPN is a rare condition, seen mainly in diabetic female patients and commonly involves the left kidney. Bilateral renal involvement is seen in $5 \%$ of the patients. ${ }^{6}$ Escherichia coli, followed by Klebsiella pneumoniae and Proteus are the common aetiological pathogens. These organisms ferment sugars within the urine and produce gases like oxygen, nitrogen, hydrogen and carbon dioxide. ${ }^{7}$ Ultrasound study and computerized tomography scan are the main imaging modalities, with $80 \%$ and $100 \%$ sensitivity respectively. ${ }^{8,9}$ The CT classification of EPN is as follows:

- Class 1: Gas confined to the collecting system;

- Class 2: Gas confined to renal parenchyma alone;

- Class 3a: Perinephric extension of gas or abscess;

- Class 3b: Extension of gas beyond the Gerota fascia;

- Class 4: Bilateral EPN or unilateral EPN with a solitary kidney. ${ }^{3}$

Based on the CT classification, the proposed therapeutic modalities are as follows:

- Class 1 and 2: antibiotic treatment combined with CTguided percutaneous drainage;

- Class 3 and 4 (extensive EPN without signs of organ dysfunction): antibiotic therapy combined with percutaneous catheter placement;

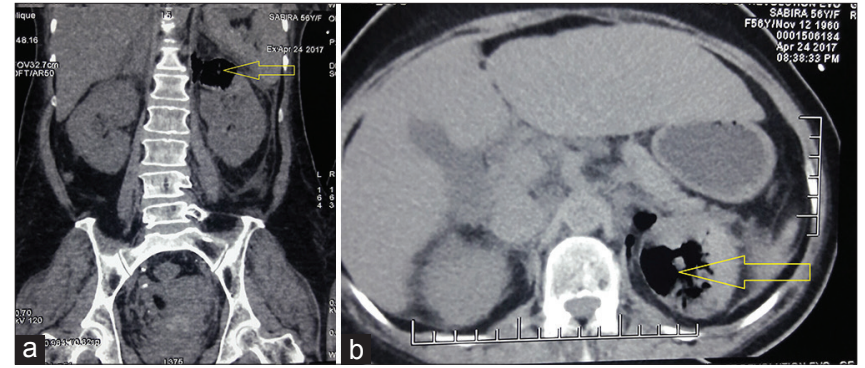

Figure 1: CT showing air in the upper pole of left kidney with thin rim of perinephric fluid (a: coronal view; b: axial view)

- Class 3 and 4 (extensive EPN with signs of organ dysfunction): nephrectomy. ${ }^{3,5}$

DHF is a life threatening condition, commonly seen following repeated dengue infections. Bleeding manifestations and plasma leakage are main features. During the early stages of the disease, the diagnosis is made by virus isolation, nucleic acid or antigen detection (NS1) while serology (IgM capture ELISA) is done at the end of the acute phase. Adequate bed rest and fluid intake and paracetamol are the main home therapies. Intravenous fluid resuscitation and platelet transfusions are given in hospitalised patients. Prophylactic platelet transfusion may be given at level of $<10,000 /$ cumm in absence of bleeding manifestations. In case of systemic massive bleeding, platelet transfusion may be needed in addition to red cell transfusion. Fresh frozen plasma or cryoprecipitate are needed in case of coagulopathy with bleeding. ${ }^{1}$ There have been reports DHF presenting with myocarditis, hepatitis, encephalopathy, glomerulonephritis and hemothorax. ${ }^{10-14}$

\section{CONCLUSION}

EPN is a rare and severe, acute necrotizing infection of the renal parenchyma and perinephric tissues with associated gas formation in the collecting system. The condition is managed with antibiotics and urological interventions like percutaneous drainage or nephrectomy. DHF is a life threatening viral infection presenting with bleeding manifestations due to thrombocytopenia and plasma leakage. Our patient is a middle aged diabetic lady who presented with symptoms of fever, myalgia, headache and dysuria, and was diagnosed to have EPN with dengue coinfection. She developed hematuria and severe thrombocytopenia; satisfying the criteria for DHF. Urological interventions were not possible because of the high risk of bleeding in the setting of DHF. She was successfully managed with broad spectrum antibiotics and platelet, packed cell and fresh frozen plasma transfusions. To the best of our knowledge, medical management of EPN, in the setting of DHF, has not been reported yet. 


\section{REFERENCES}

1. WHO. Dengue: guidelines for diagnosis, treatment, prevention and control. New edition. Geneva: World Health Organization; 2009.

2. Gupta K, Trautner BW. Urinary tract infections, pyelonephritis, and prostatitis. In: Kasper, Fauci, Hauser, Longo, Jameson, Loscalzo (ed). Harrison's Principles of Internal Medicine. $19^{\text {th }}$ ed. McGraw Hill education. pp. 861-863.

3. Huang JJ and Tseng CC. Emphysematous pyelonephritis: clinicoradiological classification, management, prognosis, and pathogenesis. Archives of Internal Medicine 2000; 160(6):797-805.

4. Wang JM, Lim HK and Pang KK. Emphysematous pyelonephritis. Scandinavian Journal of Urology and Nephrology 2007; 41(3):223-229.

5. Tseng CC, Wu JJ, Wang MC, Hor LI, Ko YH and Huang JJ. Host and bacterial virulence factors predisposing to emphysematous pyelonephritis. American Journal of Kidney Diseases 2005; 46(3):432-439.

6. Leons SMHJ and Abu Sara Y. Emphysematous pyelonephritis: case report. Kuwait Med J 2004; 36(2):134-136.

7. Huang JJ, Chen KW and Ruaan MK. Mixed acid fermentation of glucose as a mechanism of emphysematous urinary tract infection. The Journal of Urology 1991; 146(1):148-151.
8. Tang HJ, Li CM, Yen MY, Chen YS, Wann SR, Lin HH, et al. Clinical characteristics of emphysematous pyelonephritis. Journal of microbiology, immunology, and infection 2001; 34(2):125-130.

9. Ahlering TE, Boyd SD, Hamilton CL, Bragin SD, Chandrasoma PT, Lieskovsky G, et al. Emphysematous pyelonephritis: a 5-year experience with 13 patients. The Journal of Urology 1985; 134(6):1086-1088.

10. Leea IK, Leeb WH, Liua JW and Yang KD. Acute myocarditis in dengue hemorrhagic fever: a case report and review of cardiac complications in dengue-affected patients. International Journal of Infectious Diseases 2010; 14(10):919-922.

11. Mourão MPG, de Lacerda MVG, de Souza Bastos M, de Albuquerque BC and Alecrim WD. Dengue hemorrhagic fever and acute hepatitis: a case report. Brazilian Journal of Infectious Diseases 2004; 8(6):461-464.

12. Chotmongkol V and Sawanyawisuth K. Case report: Dengue hemorrhagic fever with encephalopathy in an adult. Southeast Asian J Trop Med Public Health 2004; 35(1):160-161.

13. Bhagat M, Zaki SA, Sharma S, Manglani MV. Acute glomerulonephritis in dengue haemorrhagic fever in the absence of shock, sepsis, haemolysis or rhabdomyolysis. Paediatr Int Child Health 2012;32:161-3.

14. Manappallil RG. Unprovoked hemothorax: An unusual presentation in Dengue Haemorrhagic Fever. Asian Journal of Medical Sciences 2016; 7(3):121-122.

\section{Authors contribution:}

RGM- Concept and design of case report, reviewed the literature, manuscript preparation, critical revision of manuscript and treating physician; PRM- Resident in-charge.

Orcid ID:

Dr. Robin George Manappallil: (10 http://orcid.org/0000-0003-3973-6800

Source of Support: None, Conflict of Interest: None declared. 